\title{
Quantum Hubble horizon
}

\author{
Michał Artymowski ${ }^{1,2,3}$, Jakub Mielczarek ${ }^{2,4, a}$ (i) \\ ${ }^{1}$ Institute of Theoretical Physics, Faculty of Physics, University of Warsaw, ul. Pasteura 5, 02-093 Warsaw, Poland \\ ${ }^{2}$ Institute of Physics, Jagiellonian University, Łojasiewicza 11, 30-348 Cracow, Poland \\ ${ }^{3}$ Physics Department, Ariel University, 40700 Ariel, Israel \\ ${ }^{4}$ Aix Marseille University, Université de Toulon, CNRS, CPT, Marseille, France
}

Received: 12 December 2018 / Accepted: 12 July 2019 / Published online: 29 July 2019

(C) The Author(s) 2019

\begin{abstract}
The article addresses the possibility of obtaining cosmologically relevant effects from the quantum nature of the Hubble horizon. Following the observation made by Bianchi and Rovelli (Phys Rev D 84:027502, 2011) we explore the relationship between the Planck scale discreteness of the Hubble horizon and deformations of the symmetry of rotations. We show that the so-called $q$-deformations in a natural way lead to a mechanism of condensation in the very early Universe. We argue that this provides a possible resolution of the problem of the initial homogeneity at the onset of inflation. Furthermore, we perform an analysis in terms of entropy of the quantum Hubble horizon and show that the $\Lambda \mathrm{CDM}$ model may arise from a linearly corrected Bekenstein-Hawking entropy (that is, linearly in area of the horizon). Based on this, we show that the current accelerating expansion can be associated with the entropy decrease in the Hubble volume. The results presented open new ways to explore the relation between the Planck scale effects and observationally relevant features of our Universe.
\end{abstract}

\section{Introduction}

Cosmic inflation [1-4] is a hypothetical period of the evolution of the early Universe characterized by the accelerated expansion of space. It is a powerful theoretical tool to solve problems of the classical Big Bang cosmology, such as the horizon or curvature problems. Recent observational data from the Planck satellite $[5,6]$ has set new upper bounds on the tensor-to-scalar ratio $r$, which in inflationary theories is proportional to the slow-roll parameter $\epsilon$. The upper bound for $r$ sets the GUT scale to be the maximal scale of inflation at the moment of the horizon crossing of the pivot scale, i.e. around $60 e$-folds before the end of inflation. Nevertheless,

a e-mail: jakub.mielczarek@uj.edu.pl inflation could in principle start at much higher scales, up to the Planck scale.

The idea of a high scale of the beginning of inflation is well motivated theoretically within the approach of quantum tunneling of the Universe around the Planck scale. Such a Universe is very likely to immediately recollapse unless the Planckian Universe is in the quantum state, which mimics the cosmological constant with the equation of state $p \simeq$ $-\rho$ [7-11]. The high scale of the beginning of inflation has recently also been supported by the results from the theory of causal dynamical triangulations [12]. The cosmological constant (which in the realistic case should be replaced by the inflationary potential) plays the crucial role in the process of creating a classical Universe from the "quantum foam".

The other argument to start inflation close to the quantum gravity scale is the problem of initial conditions discussed in Ref. [13], which points out that inflationary models with infinite plateaus, like Higgs [14] or Starobinsky inflation [1], require a special type of initial conditions. ${ }^{1}$ In those models the inflationary part of the potentials is limited from above by the scale of inflation, which is typically of the order of the GUT scale. The problem is the following. Let us consider a horizon in the pre-inflationary Universe filled with inflaton $\phi$, dust and radiation. We assume that at such high energies the contribution of the cosmological constant is negligible. For the plateau-like potential only the kinetic term $\dot{\phi}^{2}$ and the gradient of the field $\left(\partial^{i} \phi\right)^{2}$ have significant contributions to the inflaton's energy density. The energy density of the kinetic term, radiation, dust and gradient decrease like $a^{-6}, a^{-4}, a^{-3}$ and $a^{-2}$, respectively, where $a$ is a scale factor. Therefore,

\footnotetext{
${ }^{1}$ Note that the problem of initial conditions is still discussed within the scientific community. For opposite points of view see e.g. Refs. [15-19]. For some of the proposed solutions see Refs. [20-27]. Furthermore, in [28] the authors note that even in the absence of causality, the Starobinsky $f(R)$ model predicts local homogeneity in the pre-inflationary era. This suggests that the problem of initial conditions does not have to apply to all of the plateau models.
} 
the inhomogeneous part of the energy density shall dominate the system before the potential term of the inflaton has a chance to generate acceleration of the scale factor, which would strongly suppress inhomogeneities. As a result inflation may not be successful, unless one assumes around $10^{9}$ homogeneous, causally independent horizons at the Planck scale [13]. Models like power-law inflation or other models of chaotic inflation do not suffer from such unrealistic requirements, since in order to initiate inflation they require the existence of only one homogeneous horizon. Furthermore, as shown in Ref. [29], power-law models may have an inflationary attractor solution even for an inhomogeneous Universe. Nevertheless, power-law inflation is disfavored by the data [6], which keeps the problem of initial conditions alive.

In order to start inflation one usually assumes the existence of a patch of space which is homogeneous enough to support initial conditions for inflation. This part of the Universe would be most likely filled with a condensate of a scalar field (or fields), since beside a few exceptions cosmic inflation is usually run by a homogeneous scalar field. The creation of a homogeneous scalar field is natural on the onset of inflation, when all of the inhomogeneities are exponentially suppressed. Nevertheless, the existence of a preinflationary Planckian horizon filled with a homogeneous scalar field seems to be unlikely. In this article, we investigate the possibility of naturalness of the homogeneity of the Planckian Universe in the framework of quantum gravity.

We show that Planck scale discreteness of the cosmological (Hubble) horizon introduces a possible mechanism leading to homogeneous initial conditions at the onset of inflation. The mechanism relies on the properties of a quantum gravitational effect leading to the noncommutative behavior characterized by the so-called $q$-deformations. The values of the $q$-deformation parameters are functions of the energy density scale and in the very early Universe only a limited number of representations of the $q$-deformed group is allowed. In consequence, the Universe establishes a condensate state when the energy densities approach the Planck energy scale. The mechanism is introduced in Sect. 2, where we also stress that the $q$-deformations in combination with the Copernican principle lead to homogeneity. In Sect. 3 the meaning of the performed considerations in the context of the problem of initial homogeneity at the beginning of inflation is explained. Furthermore, a preliminary analysis of the energy density fluctuations of the Hubble horizon is performed. In Sect. 4, the entropy properties of the quantum Hubble horizon are analyzed. We show that, depending on the matter content of the Universe, entropy flow may occur either into or out of the Hubble volume. The form of the entropy as a function of the area of the Hubble sphere is reconstructed for the $\Lambda \mathrm{CDM}$ Universe. The results are summarized and discussed in Sect. 5. Furthermore, in the appendix the issue of $q$-deformations in the loop quantum gravity approach to Planck scale physics is outlined.

Throughout this article we consequently apply Planck units, where $\hbar=c=k_{B}=1$ and $G=l_{\mathrm{Pl}}^{2}$, where $l_{\mathrm{Pl}}$ denotes the Planck length.

\section{Condensation via $q$-deformations}

One of the characteristic expectations regarding the Planck scale physics is that there is a minimal length scale, being of the order of the Planck length $l_{\mathrm{Pl}} \approx 1.62 \cdot 10^{-35} \mathrm{~m}$. Depending on the particular model of Planck scale physics, the Planck length may enter in various ways into the considerations. However, the general qualitative conclusion is common-no details of the space-time structure at the scales below the Planck length can be observed. The Planck length, therefore, sets the highest (UV) limit on the resolution at which spacetime can be probed. But there is also the lowest (IR) limit which results from the causal structure of space-time and is given by the so-called Hubble radius:

$R_{\mathrm{H}}:=\frac{1}{H}$,

where $H$ is the Hubble factor. The Hubble radius allows one to define a Hubble sphere (see Fig. 1) containing all information accessible to the observer located in the center of the sphere. The sphere has the area $A_{\mathrm{H}}=4 \pi R_{\mathrm{H}}^{2}$. Because the minimal area allowed by Planckian physics is of the order of $l_{\mathrm{Pl}}^{2}$, the Hubble sphere contains approximately $A_{\mathrm{H}} / l_{\mathrm{Pl}}^{2}=4 \pi\left(R_{\mathrm{H}} / l_{\mathrm{Pl}}\right)^{2}$ elementary Planckian cells (pixels). In the present Universe, the number is extremely large:

$$
\frac{A_{\mathrm{H}}}{l_{\mathrm{Pl}}^{2}}=4 \pi\left(\frac{R_{\mathrm{H}}}{l_{\mathrm{Pl}}}\right)^{2} \approx 8 \cdot 10^{122},
$$

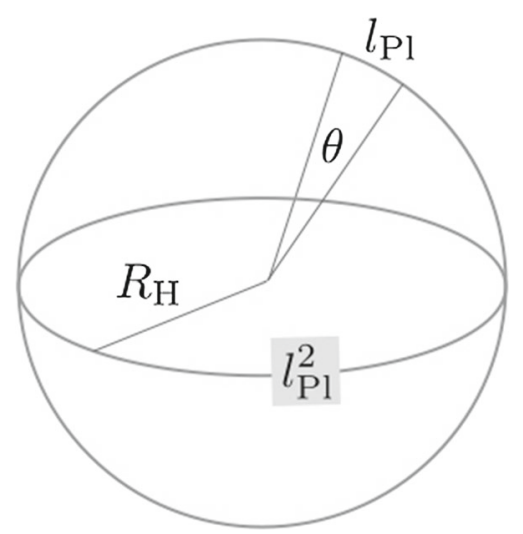

Fig. 1 Quantum Hubble horizon can be considered as a set of Planckian pixels (a single such a pixel is depicted as the shadowed square). The radius of the sphere is $R_{\mathrm{H}}$ and the maximal angular resolution is denoted $\theta$ 
where we used $R_{\mathrm{H}}=\frac{1}{H_{0}} \approx 4,4 \mathrm{Gpc}$. It is worth mentioning that the value is also proportional to the number of degrees of freedom stored at the Hubble sphere. As we will discuss later, and what will be crucial for the mechanism we are going to introduce, the quantity analyzed in Eq. (2) dramatically decreases when the Planck epoch of the evolution of the Universe if approached, where $R_{\mathrm{H}}$ falls to a value being of the order of $l_{\mathrm{Pl}}$.

As discussed in Ref. [30], the Hubble sphere decomposition on the Planckian cells leads to the maximal allowed angular resolution given by

$\theta \sim \frac{l_{\mathrm{Pl}}}{R_{\mathrm{H}}} \approx 10^{-61} \mathrm{rad}$

where the numerical value has been given for the current value of $R_{\mathrm{H}}$. Because of this, the rotational invariance is not fully satisfied but instead there is a minimal angle given by Eq. (3), below which rotations cannot be considered. In consequence, the rotation group $S O(3)$ or its double covering counterpart (the $S U$ (2) group) require an adequate modification, taking into account the maximal resolution given by Eq. (3).

Such modifications are known in mathematical physics under the name of $q$-deformations, which in the case of rotations lead to the $S U(2)_{q}$ group, where in general $q \in \mathbb{C}$. $^{2}$ In such a case the $q$-deformation factor can be written as $q=e^{i \frac{\pi}{k}}$ with $k \in\{2,3,4, \ldots\}$, which modifies properties of the $S U(2)$ group. In particular, only the following values for $j$ (labeling irreducible representations of the $S U(2)_{q}$ ) are permitted: $j \in\left\{0, \frac{1}{2}, 1, \ldots, \frac{k}{2}-1\right\}$, while the $\mathrm{SU}(2)$ group allows for arbitrary representations labeled by half-integers $j=\frac{n}{2} \in \mathbb{N}$. Furthermore, the dimensions of the irreducible representations of the $S U(2)_{q}$ group are given by the following formula:

$d_{j}^{q}=\frac{q^{2 j+1}-q^{-(2 j+1)}}{q-q^{-1}}=\frac{\sin \left(\frac{\pi}{k}(2 j+1)\right)}{\sin \left(\frac{\pi}{k}\right)}$,

so that for $q \rightarrow 1(k \rightarrow \infty)$ the $S U(2)$ case with $d_{j}=2 j+1$ is recovered.

One may ask: what is the maximal angular resolution corresponding to the case of $q$-deformation with some $j_{\max }:=$ $\frac{k}{2}-1$ ? In the case of the standard $S U$ (2) group the degeneracy of the $j$ representation is equal to $d_{j}=2 j+1$, which means that the corresponding resolution squared is $\theta^{2} \approx \frac{4 \pi}{2 j+1}$. However, in the $q$-deformed case the dimension of the representation is not a monotonic function of $j$ and the maximal degeneration corresponds to the maximum of the function (4), which is located at $j_{0}=\frac{1}{2} j_{\max }$ for which

$\overline{2 \text { The case of } q} \in \mathbb{R}$ has been introduced in [31]. $d_{j_{0}}^{q}=1 / \sin \left(\frac{\pi}{k}\right) \approx \frac{2}{\pi}\left(j_{\max }+1\right) \cdot{ }^{3}$ The approximation is valid for sufficiently large values of $j_{\max }$. Anyway, similarly to what is expected based on the formula $\theta^{2} \approx \frac{4 \pi}{2 j+1}$, the resolution squared is $\theta^{2} \sim \frac{1}{j_{\max }+1}$. In consequence, the $\pi / k$ factor entering the expression $q=e^{i \frac{\pi}{k}}$ can be written as

$\frac{\pi}{k}=\frac{\pi}{2\left(j_{\max }+1\right)} \sim \theta^{2} \sim \frac{l_{\mathrm{P} 1}^{2}}{A_{\mathrm{H}}}$,

where we employed the formula for the maximal angular resolution (3). Furthermore, from the definition, the area of the Hubble sphere is proportional to the inverse square of the Hubble factor and, based on the Friedmann equation, $H^{2}=$ $\frac{8 \pi l_{\mathrm{Pl}}^{2}}{3} \rho$, we can write $\frac{\pi}{k} \sim \rho / \rho_{\mathrm{Pl}}$, where the Planck energy density $\rho_{\mathrm{Pl}}:=l_{\mathrm{Pl}}^{-4}$. Therefore, the $q$-deformation parameter can be written as

$q=\exp \left(i \frac{\pi}{2} \frac{\rho}{\rho_{*}}\right)$

where $\rho$ is the total energy density of the Universe (including cosmological constant) and $\rho_{*} \sim \rho_{\mathrm{Pl}}$ is an energy scale comparable with the Planck energy density. Equation (6) is defined such that $\rho_{*}$ is the maximal energy density at which $q=i$ and consequently $k=2$ and $j_{\max }=0$. The angular maximal resolution square tends to $4 \pi$ in this limit, which corresponds to full isotropy.

From the above arguments one can now conclude that because of the Planck scale discreteness the rotational symmetry is affected and the magnitude of these effects increases with the increase of the energy density in the Universe. While the effect is marginal today, when the energy density reaches Planck values the angular resolution decreases dramatically, which is associated with the reduction of the allowed representations of the group of rotations. In the quantum case, the dimension of the Hilbert space associated with the $S U(2)_{q}$ invariant system decreases with the increase of the energy density. In the limit when of $\rho=\rho_{*}$, only the $j=0$ state $|0\rangle$ is permitted.

Therefore, if gravitational or matter degrees of freedom are associated with the angular momentum (spin), such a system undergoes condensation as a result of the $q$-deformation, which prevents excited states from being occupied. In the $q \rightarrow i$ limit the quantum state of multiple degrees of freedom quantum system reduces to the ground state:

$|0\rangle \otimes|0\rangle \otimes|0\rangle \otimes|0\rangle \otimes|0\rangle \otimes|0\rangle \otimes \cdots$.

The decrease of the angular resolution naturally indicates that the configuration is becoming isotropic. Furthermore, taking

\footnotetext{
${ }^{3}$ In Ref. [30] it has been argued that $\theta^{2} \approx 2 / j_{\max }$.
} 
(a)

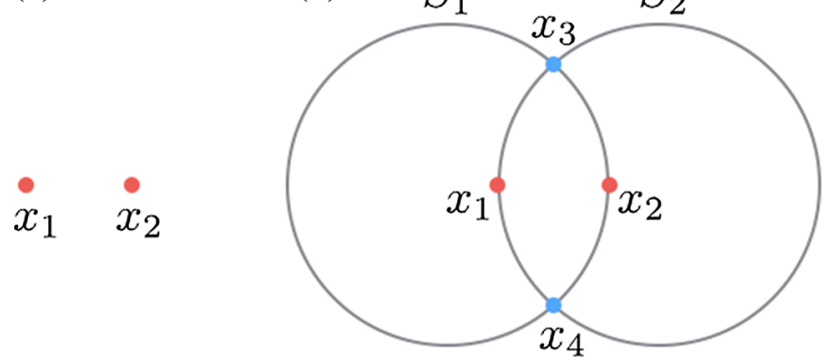

Fig. 2 a Arbitrarily chosen points $x_{1}$ and $x_{2}$ separated by $d\left(x_{1}, x_{2}\right)<$ $2 R_{\mathrm{H}}$. b Geometric constructions used to prove that $\left\langle\phi\left(x_{1}\right)\right\rangle=\left\langle\phi\left(x_{2}\right)\right\rangle$ assuming statistical isotropy and the Copernican cosmological principle

into account the Copernican cosmological principle (no point in space is distinguished), the isotropy implies homogeneity:

$$
\begin{aligned}
& \text { Isotropy }+ \text { Copernican cosmological principle } \\
& \Rightarrow \text { homogeneity. }
\end{aligned}
$$

For completeness of our considerations let us give a simple proof of the above statement. For this purpose let us consider two points $x_{1}$ and $x_{2}$ separated by $d\left(x_{1}, x_{2}\right)<2 R_{\mathrm{H}}$ (see Fig. 2) and some field $\phi(x)$ which is probed. The task is to prove that, for any such two points, the statistical isotropy and Copernican cosmological principle imply that $\left\langle\phi\left(x_{1}\right)\right\rangle=$ $\left\langle\phi\left(x_{2}\right)\right\rangle$. The averaging is performed either over an ensemble of the configurations of the field $\phi(x)$ or over different points.

The proof can be conducted with the use of following geometrical construction: Let us consider two Hubble spheres $S_{1}$ and $S_{2}$ as shown in Fig. 2. The statistical isotropy implies that $\left\langle\phi\left(x_{3}\right)\right\rangle=\left\langle\phi\left(x_{4}\right)\right\rangle$ for both observers located at the centers of the Hubble spheres. Now, because $\forall x \in S_{1}$ we have $\langle\phi(x)\rangle=\left\langle\phi\left(x_{3}\right)\right\rangle=\left\langle\phi\left(x_{4}\right)\right\rangle$, in particular $\left\langle\phi\left(x_{2}\right)\right\rangle=\left\langle\phi\left(x_{3}\right)\right\rangle=\left\langle\phi\left(x_{4}\right)\right\rangle$. On the other hand, $\forall x \in S_{2}$ we have $\langle\phi(x)\rangle=\left\langle\phi\left(x_{3}\right)\right\rangle=\left\langle\phi\left(x_{4}\right)\right\rangle$, which implies that $\left\langle\phi\left(x_{1}\right)\right\rangle=\left\langle\phi\left(x_{3}\right)\right\rangle=\left\langle\phi\left(x_{4}\right)\right\rangle$. Combining these two observations it is straightforward to infer that $\left\langle\phi\left(x_{1}\right)\right\rangle=\left\langle\phi\left(x_{2}\right)\right\rangle$. In the case when two points are separated by $d\left(x_{1}, x_{2}\right) \geq 2 R_{\mathrm{H}}$, auxiliary intermediate points shall be introduced and a sequence of inferences of the kind presented above has to be made. This completes the proof.

Therefore, when all degrees of freedom are placed in the same ground state, the corresponding configuration of space is expected to be ideally homogeneous. This is, of course, under the assumption that the angular momentum variables associated with the rotational invariance play a significant role in the description of the quantum state of the gravitational field. As discussed in the appendix, this is the case at least in one of the most promising approaches to quantum gravity.

\section{Initial conditions for inflation}

The considerations presented in the previous section assumed that the Hubble horizon is a sphere, which is the case for isotropic cosmological models. However, in the case of anisotropic cosmologies the geometry of a horizon in no longer a sphere. In the particular case of the Bianchi I Universe, the Hubble horizon generalizes to a spheroid geometry with the semi-axes $R_{\mathrm{H}, i}:=1 / H_{i}$, where $H_{i}:=\frac{\dot{a}_{i}}{a_{i}}$ are the Hubble factors associated with the three different scale factors $a_{i}$, with $i=1,2,3$. In consequence, the Hubble volume is $V_{\mathrm{H}}=\frac{4}{3} \pi a_{1} a_{2} a_{3}$ and the area of the Hubble horizon is no longer given by the formula $A_{\mathrm{H}}=4 \pi R_{\mathrm{H}}^{2}$. Therefore, it is relevant to consider whether the mechanism presented in the previous section is spoiled by cosmological anisotropy.

It is worth stressing that the observed Universe does not exhibit a statistically significant deviation from anisotropy at the cosmological scales [32]. In consequence, the assumption of isotropy of inflationary and post-inflationary Universe (as we made in Sect. 4) is well justified. However, theoretical considerations of the generic cosmological singularity related to the Belinsky-Khalatnikov-Lifschitz (BKL) conjecture $[33,34]$ suggest that anisotropy may play an important role in the very early Universe. The BKL conjecture indicates that a near-singularity Universe is characterized by ultralocal and strongly anisotropic chaotic evolution. Furthermore, only stiff mater contributes non-trivially to the cosmological dynamics.

For the purpose of our discussion, let the anisotropies predicted by the BKL begin to have a dominant effect above the energy density $\rho_{\mathrm{BKL}}$. Furthermore, let $\rho_{\mathrm{I}}$ denote the energy density at the onset of inflation. The usual assumption is that the energy scale of inflation $\rho_{\mathrm{I}}$ is smaller than the energy scale of the BKL phase $\rho_{\mathrm{BKL}}$. In particular, this concerns the discussion presented in Refs. $[13,20]$. In this case ${ }^{4}$, which is simpler and which is not ruled out by the cosmological data, the following three possibilities can be considered:

(i) $\rho_{\mathrm{I}}>\rho_{\mathrm{Pl}}$. In this case both the energy density at the onset of inflation and the BKL phase are beyond the Planck scale. In such a case, there is no semi-classical counterpart to the BKL phase, which is just an artifact of classical theory. In consequence, in this case there is no reason to take the BKL anisotropy into account when the mechanism introduced in Sect. 2 is considered.

(ii) $\rho_{\mathrm{BKL}}>\rho_{\mathrm{Pl}}>\rho_{\mathrm{I}}$. As in (i), in this case there is no semiclassical counterpart of the anisotropic BKL phase. This case is consistent with the assumptions made in Refs. $[13,20]$.

(iii) $\rho_{\mathrm{Pl}}>\rho_{\mathrm{BKL}}$. In this case the pre-inflationary epoch may be characterized by the BKL-type anisotropy, and

\footnotetext{
${ }^{4}$ Which is both simpler and not ruled out by the cosmological data.
} 
the formulas derived in Sect. 2 cannot be directly applied. In this case, corrections originating from cosmological anisotropy have to be taken into account. If the anisotropy is not a leading effect, the area of the Hubble horizon is approximately given by $A_{\mathrm{H}} \approx 4 \pi \sqrt[p]{\frac{\left(R_{\mathrm{H}, 1} R_{\mathrm{H}, 2}\right)^{p}+\left(R_{\mathrm{H}, 1} R_{\mathrm{H}, 3}\right)^{p}+\left(R_{\mathrm{H}, 2} R_{\mathrm{H}, 3}\right)^{p}}{3}}$, where $p \approx 1.6075$. Furthermore, it is possible to apply the $S U(2)_{q}$ group by considering coordinates rescaled by the semi-axes $R_{\mathrm{H}, i}$ (for Bianchi I). This suggests that at least the qualitative conclusions of Sect. 2 are preserved when some anisotropy is present. However, further detailed investigations are needed to make definitive conclusions.

In what follows we will focus on the cases (i) and (ii) in which the BKL anisotropy is not taken into account. Under this assumption, and using Eq. (6), cosmological evolution associated with the transition $q \approx i$ (UV) $\rightarrow q=1$ (IR), can be considered. The scenario is related with decondensation in which the value of $j_{\max }$ increases from $j_{\max }=0$ to $j_{\max } \rightarrow \infty$. In consequence, new quantum states are "released" together with the growth of the scale factor and decrease of the energy density.

An interesting issue to consider is if the described mechanism can provide proper initial conditions at the beginning of inflation, which usually requires a huge order of homogeneity (see e.g. Refs. $[13,20]$ ). Let us discuss this issue in more detail. We denote by $t_{\mathrm{I}}$ the time at which inflation starts and $t_{\mathrm{Pl}}$ corresponds to the Planck epoch, in which $q \rightarrow i$. The value of the Hubble factor at the beginning of inflation is $H\left(t_{\mathrm{I}}\right)$ and the associated value of the Hubble radius $R_{\mathrm{H}}\left(t_{\mathrm{I}}\right)=1 / H\left(t_{\mathrm{I}}\right)$. For the inflation to start the homogeneity scale $L$ at the beginning of inflation $L\left(t_{\mathrm{I}}\right)$ must satisfy $L\left(t_{\mathrm{I}}\right) \geq R_{\mathrm{H}}\left(t_{\mathrm{I}}\right)$. The homogeneity scale at $t_{\mathrm{I}}$ and $t_{\mathrm{Pl}}$ can be related via $L\left(t_{\mathrm{I}}\right)=L\left(t_{\mathrm{Pl}}\right) \frac{a\left(t_{\mathrm{I}}\right)}{a\left(t_{\mathrm{P}}\right)}$, which leads to the condition

$L\left(t_{\mathrm{Pl}}\right) \geq \frac{a\left(t_{\mathrm{Pl}}\right)}{a\left(t_{\mathrm{I}}\right)} \frac{H\left(t_{\mathrm{Pl}}\right)}{H\left(t_{\mathrm{I}}\right)} R_{\mathrm{H}}\left(t_{\mathrm{Pl}}\right)$,

with $R_{\mathrm{H}}\left(t_{\mathrm{Pl}}\right) \approx l_{\mathrm{Pl}}$. For the barotropic matter, the above inequality can be written as

$L\left(t_{\mathrm{Pl}}\right) \geq\left(\frac{\rho_{\mathrm{Pl}}}{\rho_{\mathrm{I}}}\right)^{\frac{1+3 w}{6(1+w)}} l_{\mathrm{Pl}}$,

where $w$ is the barotropic index. The problem of the initial homogeneity is associated with the fact that for the preinflationary period (where $1+3 w>0$ ) and $\rho_{\mathrm{Pl}} \gg \rho_{\mathrm{I}}$ we have $L\left(t_{\mathrm{Pl}}\right) \gg l_{\mathrm{Pl}}$. The homogeneity scale at the Planck epoch has to be much bigger than the Planck length (or equivalently the Hubble radius). Due to the condensation mechanism introduced in Sect. 2 such a condition has, however, a chance to be satisfied. Even if one does not support the criticism of
Ref. [13], the initial homogeneity of the Universe can be still considered as an indication of the naturalness of the inflationary paradigm. We would also like to emphasize that our approach is independent of the particular model of inflation.

Furthermore, some preliminary considerations regarding primordial perturbations can be made. Let us namely notice that due to the "Planckian pixels", the number of degrees of freedom associated with the Hubble sphere is roughly

$N \approx \frac{A_{\mathrm{H}}}{l_{\mathrm{Pl}}^{2}}=4 \pi \frac{R_{\mathrm{H}}^{2}}{l_{\mathrm{Pl}}^{2}}$.

Assuming the equilibrium configuration, the average energy is

$\langle E\rangle=N \frac{1}{2} T=\frac{A_{\mathrm{H}} T}{2 l_{\mathrm{Pl}}^{2}}$.

This allows us to quantify the thermal fluctuations of the energy:

$\sigma_{E}^{2}=\langle E\rangle^{2}-\left\langle E^{2}\right\rangle=T^{2} \frac{\partial\langle E\rangle}{\partial T}=\frac{A_{\mathrm{H}} T^{2}}{2 l_{\mathrm{Pl}}^{2}}$.

In consequence, the relative fluctuations of the energy of the Hubble sphere are

$\delta_{E}:=\frac{\sigma_{E}}{\langle E\rangle} \sim \frac{1}{\sqrt{N}} \sim \frac{H}{m_{\mathrm{Pl}}}$.

Considering the energy density $\rho=\frac{E}{V_{\mathrm{H}}}$ in a fixed Hubble volume $V_{\mathrm{H}}$ we obtain

$\delta_{\rho}:=\frac{\sigma_{\rho}}{\langle\rho\rangle} \sim \frac{H}{m_{\mathrm{Pl}}}$,

and the power of the perturbations

$\left|\delta_{\rho}\right|^{2} \sim\left(\frac{H}{m_{\mathrm{Pl}}}\right)^{2}$

The predicted amplitude of the fluctuations is, therefore, in qualitative agreement with the inflationary power spectrum. However, the result is very preliminary and further more sophisticated investigations are required to confirm if the correct inflationary power spectrum can be recovered. In particular, as shown in Ref. [35], considerations similar to the one presented above may lead to a nearly scale invariant spectrum of primordial perturbations.

\section{Thermodynamics of the Hubble horizon}

As we have discussed, the $q$-deformations can be interpreted as a consequence of the finite angular resolution associated with the Planck scale "pixels" at the Hubble sphere. The 
(a) Black hole

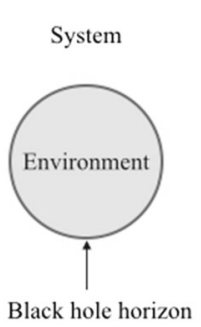

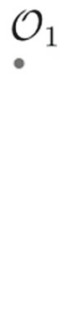

(b)

Universe

Environment

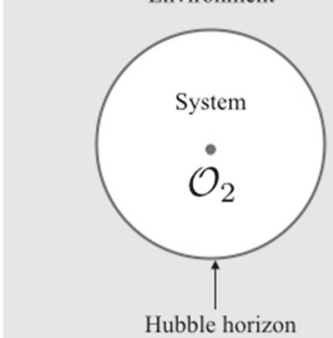

Fig. 3 a For an observer $\mathcal{O}_{1}$, the black hole entropy is a measure of the lack of knowledge about the environmental degrees of freedom, hidden under the black hole horizon. b In cosmology, for any observer $\mathcal{O}_{2}$ there is always a Hubble horizon, which defines he boundary between the system (interior of the Hubble sphere) and the environment (exterior of the Hubble sphere)

quantum nature of the Hubble sphere indicates that there is a finite entropy associated with the area of the Hubble sphere [36-38]. The entropy is a measure of the observer's lack of information about the state of environment. From the definition, the environmental degrees of freedom are those which are inaccessible to the observer. In the cosmological context, the interior of the Hubble sphere can be called a system and the region outside of the Hubble radius is the environment. The situation is quite the opposite to the case of black holes, where the Bekenstein-Hawking entropy $[39,40]$,

$S_{\mathrm{BH}}=\frac{1}{4} \frac{A}{l_{\mathrm{Pl}}^{2}}$

is associated with lack of access to the information stored inside the black hole horizon for an observer located outside of the black hole (see Fig. 3).

Following the holographic principle [41], let us initially assume that the entropy associated with the Hubble horizon is the same as in the case of the black hole. At the microscopic level the entropy may be explained by relating elementary degrees of freedom with every Planck scale "pixel" in the spirit of the it from bit conjecture [42]. The number of such elementary Planck cells is $N \approx A / l_{\mathrm{Pl}}^{2}$. Assuming that state of a Planck cell is encoded by a single bit, the number of microstates corresponding to the configuration with a fixed area is given by $\Omega=2^{N}$. Based on this, the Boltzmann entropy of the horizon is $S=\ln \Omega=N \ln 2 \sim A / l_{\mathrm{Pl}}^{2}$.

Let us now consider the system to be defined as the interior of the Hubble sphere of the radius $R_{\mathrm{H}}$ and the environment to be the exterior (see Fig. 3). The corresponding entropy associated with the horizon can then be expressed as

$S_{\mathrm{H}}=s S_{\mathrm{BH}}=\frac{s}{4} \frac{4 \pi R_{\mathrm{H}}^{2}}{l_{\mathrm{Pl}}^{2}}=\frac{s}{4} \frac{4 \pi}{l_{\mathrm{Pl}}^{2} H^{2}}$

where we introduced $s=\{-1,0,1\}$. The choice of the factor $s$ depends on the direction of the entropy transfer between the system and environment. In the case of the black holes (for an observer located outside of the horizon) the increase of the horizon area is associated with the increase of the entropy of the environment (the volume outside of the horizon is decreasing). The entropy is transferred from the region of decreasing volume to the region of increasing volume.

In the cosmological context, it is worth to consider the ratio between the Hubble radius $R_{\mathrm{H}}$ and some physical scale $L \propto a$ :

$$
\frac{R_{\mathrm{H}}}{L} \sim \frac{1}{a|H|} \sim|H|^{-\frac{1+3 w}{3(1+w)}} .
$$

In the expanding Universe, for $w>-\frac{1}{3}$, the horizon size is increasing with respect to the physical scales. We, therefore, expect that the entropy of the Hubble volume is increasing and in consequence $s=1$. Simply stated, more degrees of freedom enter to the system so $d S_{\mathrm{H}}>0$. In turn, for $w<-\frac{1}{3}$ the Hubble radius is shrinking with respect to physical scales and the entropy is decreasing, therefore $s=-1$. Here, the degrees of freedom escape from the system leading to $d S_{\mathrm{H}}<$ 0 . For $w=-\frac{1}{3}$ we have $\frac{R_{\mathrm{H}}}{L}=$ const and in consequence we have to fix $s=0$, since there is no entropy transfer in this case. Furthermore, the volume enclosed by the Hubble sphere is $V_{\mathrm{H}}=\frac{4}{3} \pi R_{\mathrm{H}}^{3}$, which allows one to write the energy in this volume as $U=V_{\mathrm{H}} \rho$, where $\rho$ is the energy density.

The system under consideration (the interior of the Hubble sphere) satisfies the first law of thermodynamics,

$\mathrm{d} U=T \mathrm{~d} S_{\mathrm{H}}-p \mathrm{~d} V_{\mathrm{H}}$,

and the second law of thermodynamics,

$\mathrm{d} S_{\mathrm{H}}+\mathrm{d} S_{\text {env }} \geq 0$,

where $\mathrm{d} S_{\text {env }}$ is the entropy change of the environment. The weak inequality reduces to equality if irreversible processes are not present in the system. In particular, if the entropy change is only by the exchange of heat between the system and environment, then $\mathrm{d} Q_{\mathrm{H}}=-\mathrm{d} Q_{\text {env }}$ and in consequence $\mathrm{d} S_{\mathrm{H}}+\mathrm{d} S_{\text {env }}=0$. In such a case the entropy of the system $\left(S_{\mathrm{H}}\right)$ can be reduced to the cost of an increase of the entropy of the environment $\left(S_{\mathrm{env}}\right)$. Such a behavior is one of the characteristics of the open systems, which allows for departure from the state of thermal equilibrium [43]. In what follows, we will focus on the case of the entropy exchange by the heat transfer, such that the entropy (18) can be used for both the system and the environment (but with opposite signs).

Furthermore, the two laws of thermodynamics are accompanied by the equation of state (EOS), which in the case 
considered is the Friedmann equation:

$H^{2}=\frac{8 \pi l_{\mathrm{Pl}}^{2}}{3} \rho$,

where we neglected the curvature term. Using the expression for the area of the Hubble horizon $A=4 \pi / H^{2}$, the Friedmann EOS can be written as

$\frac{3}{2 l_{\mathrm{Pl}}^{2}}=A \rho$.

Applying this EOS to the first law of thermodynamics (20), together with $U=V_{\mathrm{H}} \rho=\frac{1}{4 l_{\mathrm{Pl}}^{2}} \sqrt{\frac{A}{\pi}}$, the expression for the derivative of $S_{\mathrm{H}}$ can be obtained:

$\mathrm{d} S_{\mathrm{H}}=\frac{\sqrt{A}}{4 \sqrt{\pi} T}\left(\frac{1}{2 l_{\mathrm{Pl}}^{2} A}+p\right) \mathrm{d} A$,

which shows that $S_{\mathrm{H}}$ can be written as a function of a single variable $A$. It remains to express $T$ and $p$ in terms of $A$. For this purpose, let us rewrite the first law of thermodynamics (20) into the form

$\dot{\rho}-3 \frac{\dot{H}}{H}(\rho+p)=\frac{T}{V_{\mathrm{H}}} \frac{\mathrm{d} S_{\mathrm{H}}}{\mathrm{d} t}$.

Note that this equation is significantly different from the case in which one considers the thermodynamics of a local, unspecified region of the Universe. In our case, Eq. (25) is not equivalent to the continuity equation. On the other hand, the energy density $\rho$ satisfies the local conservation laws, which lead to the continuity equation,

$\dot{\rho}+3 H(\rho+p)=0$.

Equations (25) and (26) may be re-written as

$3 H(\rho+p) \frac{\rho+3 p}{2 \rho}=\frac{T}{V_{\mathrm{H}}} \frac{\mathrm{d} S_{\mathrm{H}}}{\mathrm{d} t}$.

In agreement with our previous analysis, Eq. (27) predicts that $d S_{\mathrm{H}}=$ const for $p=-\frac{1}{3} \rho$ but also for $p=-\rho$ due to the fact that the entropy is a function of $A$, which remains constant in the de Sitter Universe.

With the use of the Friedmann equation (22) and employing (18) we can now solve Eq. (27) such that the expression for the pressure can be found:

$p=-\frac{1}{3} \rho+\frac{s T H}{2 l_{\mathrm{Pl}}^{2}}$.

This equation implies that in the expanding Universe with $w>-\frac{1}{3}(s=1)$ the pressure $p>-\frac{1}{3} \rho$, as expected. On the other hand, for $w<-\frac{1}{3}(s=-1)$ we have $p<-\frac{1}{3} \rho$. The cosmic acceleration can therefore be associated with a decrease of the entropy of the horizon. More specifically, accelerated expansion and the decrease of entropy are tautology for the Hubble horizon entropy given by the BekensteinHawking formula with negative sign.

Taking the barotropic equation of state $p=w \rho$, the expression for the temperature of the thermal bath (environment) can be found,

$T=\frac{(3 w+1)}{2 s} \frac{H}{2 \pi}$.

One can notice that for the de Sitter case $(w=-1$ and $s=-1$ ) the well-known expression for the de Sitter horizon temperature $T=\frac{H}{2 \pi}[44]$ is correctly recovered.

The above considerations concerned the case of a barotropic fluid. But to be more realistic, let us now study the $\Lambda \mathrm{CDM}$ cosmology and try to reconstruct the expression for the entropy function. In this case, the total energy density is a sum of contributions from (pressureless) dark matter and the cosmological constant $\Lambda$ :

$\rho=\rho_{D M}+\rho_{\Lambda}=\frac{\rho_{D M, 0}}{a^{3}}+\frac{\Lambda}{8 \pi G}$.

The contribution of radiation has been neglected. However, such a fraction may occur, in particular due to the cosmological analog of Hawking radiation. Meanwhile, such a contribution is expected to be rather marginal; situations when the cosmological Hawking radiation may play a significant role cannot be ruled out (see Ref. [45]).

Applying Eqs. (30) and (25) we find that

$\frac{\mathrm{d} S_{\mathrm{H}}}{\mathrm{d} A}=\frac{1}{16 \pi l_{\mathrm{Pl}}^{2}} \frac{H}{T}\left(1-\frac{\Lambda}{H^{2}}\right)$.

In the case of the de Sitter Universe, with $T=\frac{H}{2 \pi}$ and $H^{2}=$ $\frac{\Lambda}{3}$, Eq. (31) gives

$\frac{\mathrm{d} S}{\mathrm{~d} A}=-\frac{1}{4 l_{\mathrm{Pl}}^{2}}$,

which correctly leads to the expression

$S_{\mathrm{H}}=S_{0}-\frac{A}{4 l_{\mathrm{Pl}}^{2}}$.

Note that the minus sign in this equation corresponds to $s=$ -1 in Eq. (18). In the general case, we do not know what the relation between $T$ and $H$ is. However, for dimensional reasons, we expect that the linear relation is preserved such that $T=c H$, with some dimensionless constant $c$. Using this, Eq. (31) can be written as 
$\frac{\mathrm{d} S_{\mathrm{H}}}{\mathrm{d} A}=\frac{1}{16 \pi l_{\mathrm{Pl}}^{2} c}\left(1-\frac{\Lambda}{4 \pi} A\right)$

which can be solved,

$S_{\mathrm{H}}=S_{0}+\frac{A}{4 l_{\mathrm{Pl}}^{2}} \frac{\left(1-\frac{\Lambda}{8 \pi} A\right)}{4 \pi c}$.

In the $\Lambda \rightarrow 0$ limit the expression correctly reduces to the dust case $(w=0)$ for which $S_{\mathrm{H}}$ is given by Eq. (18) with $s=1$ and $c=\frac{1}{4 \pi}$ [from Eq. (29)]. The de Sitter limit is a little more tricky, since in this case the entropy is a constant because the area of the horizon $A=\frac{12 \pi}{\Lambda}$. This can be taken into account by choosing the integration constant $S_{0}$ in Eq. (35) to be

$S_{0}=\frac{9 \pi}{4 l_{\mathrm{Pl}}^{2} \Lambda}$

This guarantees that in the de Sitter limit the entropy (35) correctly reduces to the Bekenstein-Hawking entropy of de Sitter space, with negative sign (as expected for $w=-1$ ), i.e.

$S_{d S}=-\frac{A}{4 l_{\mathrm{Pl}}^{2}}=-\frac{3 \pi}{l_{\mathrm{Pl}}^{2} \Lambda}$.

There is, however, a problem with the $S_{0}$ given by Eq. (36): this factor diverges in the $\Lambda \rightarrow 0$ limit. But one has to keep in mind that what physically matters is not the absolute value of entropy but the entropy change, which is the subject of measurements. The entropy change is always well defined and the issue with the limit $\Lambda \rightarrow 0$ does not spoil the behavior of the entropy change expected in this limit. This is because the derivative of $S_{\mathrm{H}}$ can be always taken before the $\Lambda \rightarrow 0$ limit.

Equation 35 indicates that the $\Lambda \mathrm{CDM}$ model can be seen as a result of the thermodynamics of the Hubble volume with entropy given by the linearly corrected BekensteinHawking entropy. It is worth stressing that the finiteness of the entropy is a consequence of the Planck scale discreteness of the Hubble horizon. Furthermore, corrections to the Bekenstein-Hawking entropy arise in various approaches to quantum gravity, such as loop quantum gravity [46]. However, the corrections are typically of the logarithmic type, and the agreement of the entropy (35) with some models of the quantum gravitational degrees of freedom is to be examined.

Moreover, based on Eq. (34), one can conclude that the entropy of the system (Hubble volume) is decreasing for the $\Lambda$ CDM Universe if

$\Lambda>\frac{4 \pi}{A}=H^{2}$.
Based on the most up to date astronomical observations [47] we have

$\Omega_{\Lambda}:=\frac{\Lambda}{3 H_{0}^{2}} \approx 0.69 \pm 0.01$,

where $H_{0}$ is the current value of the Hubble factor, which gives

$\Lambda \approx 2.07 H_{0}^{2}>H_{0}^{2}$.

The condition (38) is, therefore, satisfied in the observed Universe, allowing for the entropy decrease. The presented results suggest that there is relation between the cosmic accelerated expansion and the entropy reduction (complexity growth) in the observable Universe. This possibility will be investigated in more detail elsewhere.

\section{Summary}

In this article, we have performed an analysis of possible cosmologically relevant consequences of the Planck scale discreteness of the Hubble horizon. Following the results presented in Ref. [30], we have associated the quantum nature of the Hubble horizon with deformation of the rotation symmetry. Mathematically, this relationship leads to the so-called $q$-deformations of the $S O(3)$ or $S U(2)$ groups. Using the fact that the $q$-deformation leads to the constraint on the number of irreducible representations, we have shown that in the limit of Planckian energy densities only the ground states can be occupied. This provides a mechanism of generation of primordial isotropy. Then, combining this with the Copernican cosmological principle, we argued that the homogeneity spanned over many Hubble volumes at the Planck epoch can be obtained. This gives a possible resolution of the problem of the initial homogeneity at the onset of inflation.

The discreteness of the Hubble horizon leads to a finite entropy function associated with heat exchange across the horizon. In particular, the entropy function may take the form of the Bekenstein-Hawking formula. In our studies, we performed a thermodynamical analysis of the system defined as the interior of the Hubble volume and the exterior playing the role of environment. We have shown that the entropy transfer can take place in both directions between the system and the environment. The Hubble volume can, therefore, be interpreted as an open system. In the expanding Universe, for the barotropic index $w>-\frac{1}{3}$ the system gains entropy from the environment. On the other hand, for $w<-\frac{1}{3}$ the system is reducing its entropy. The latter case may have profound consequences for the increase of complexity in the observed Universe (to be compared with Ref. [48]). Furthermore, there is no entropy (heat) transfer for $w=-\frac{1}{3}$. Finally, 
we have reconstructed the form of the entropy function for the $\Lambda \mathrm{CDM}$ model, obtaining the Bekenstein-Hawking formula with a correction linear in the area of the horizon. Such corrections may possibly arise due to more detailed counting of quantum states associated with the cosmological horizon. The results open new ways to explore the relation between the Planck scale effects and observationally relevant features of our Universe.

Acknowledgements MA was supported by the Iuventus Plus Grant no. 0290/IP3/2016/74 from the Polish Ministry of Science and Higher Education. JM is supported by the Grant DEC-2014/13/D/ST2/01895 of the National Centre of Science and by the Mobilność Plus Grant 1641/MON/V/2017/0 of the Polish Ministry of Science and Higher Education

Data Availability Statement This manuscript has no associated data or the data will not be deposited [Authors' comment: Experimental data was not used.]

Open Access This article is distributed under the terms of the Creative Commons Attribution 4.0 International License (http://creativecomm ons.org/licenses/by/4.0/), which permits unrestricted use, distribution, and reproduction in any medium, provided you give appropriate credit to the original author(s) and the source, provide a link to the Creative Commons license, and indicate if changes were made. Funded by SCOAP ${ }^{3}$.

\section{Appendix: $q$-Deformation in loop quantum gravity}

One of the most studied approaches to the Planck scale physics is the background independent loop quantum gravity (LQG). The starting point for LQG is the formalism of the Ashtekar variables for which the $A$ and $E$ are canonical fields, satisfying the $\mathfrak{s u}(2)$ algebra, are considered [49]. The phase space of such a classical GR written in the framework of Ashtekar variables is affine. However, while passing to LQG, the connection $A$ is a subject of exponentiation, forming a holonomy which is an element of the compact group $\mathrm{SU}$ (2) [50]. The fluxes constructed with the use of $E$ are elements of the $\mathfrak{s u}(2)$ algebra.

In the covariant formulation, the LQG is related to the so-called Ponzano-Regge model of quantum gravity [51], which relies on the $\mathrm{SU}(2)$ group. As has been shown for the 2+1-dimensional Ponzano-Regg models, an unbounded value of $j$ leads to the IR divergences called spikes. At the beginning of the 1990s, it has been shown first at the level of purely mathematical considerations (Turaev-Vito model) and then in the work of Mizoguchi and Tada [52] that the divergences can be cured if the $q$-deformation of the SU(2) group is introduced. It was concluded that, if a deformation with $q \neq 1$ is present, the values of $j$ are bounded from above, removing the IR divergences of the theory. Moreover, in the $(2+1)$-dimensional case it has been shown that the deformation parameter $q$ introduces non-vanishing cosmo- logical constant into the theory. Namely, one can find that the value of the cosmological constant $\Lambda$ is related to the parameter $q$ via the formula

$q=e^{i \Lambda l_{\mathrm{Pl}}^{2}}$

so that for $\Lambda \rightarrow 0$ the undeformed case is recovered. It is unknown, however, if this relation holds in the $(3+1)$ dimensional case [53]. Taking the current value of the Hubble radius $\sim 1 / \sqrt{\Lambda} \sim 10^{26} \mathrm{~m}$ one obtains $q \simeq 1+i 10^{-122}$ and $j_{\max } \sim 10^{122}$.

It is worth stressing that Eq. (41) is consistent with Eq. (6). Namely, the energy density of the cosmological constant is

$\rho_{\Lambda}=\frac{\Lambda}{8 \pi l_{\mathrm{Pl}}^{2}}$,

which, when applied to Eq. (6), reproduces Eq. (41).

\section{References}

1. A.A. Starobinsky, Phys. Lett. B 91, 99 (1980)

2. A.H. Guth, Phys. Rev. D 23, 347 (1981)

3. D.H. Lyth, A. Riotto, Phys. Rept. 314, 1 (1999)

4. A.R. Liddle, New Astron. Rev. 45, 235 (2001)

5. P.A.R. Ade et al. [Planck Collaboration], Astron. Astrophys. 571, A16 (2014)

6. Y. Akrami et al. [Planck Collaboration], Planck 2018 results. X. Constraints on inflation. arXiv:1807.06211 [astro-ph.CO]

7. E.B. Gliner, Sov. Phys. JETP 22, 378 (1966)

8. R. Brout, F. Englert, E. Gunzig, Gen. Rel. Grav. 10, 1 (1979)

9. L.P. Grishchuk, Y.B. Zeldovich, Complete Cosmological Theories, in Quantum Gravity, ed. by M.A. Markov, P.C. West, Springer, Boston, MA (1984)

10. A. Vilenkin, Phys. Lett. 117B, 25 (1982)

11. V. Mukhanov, Fortschr. Phys. 63, 36 (2015)

12. J. Ambjorn, A. Goerlich, J. Jurkiewicz, R. Loll, Phys. Rept. 519, 127 (2012)

13. A. Ijjas, P.J. Steinhardt, A. Loeb, Phys. Lett. B 723, 261 (2013)

14. F.L. Bezrukov, M. Shaposhnikov, Phys. Lett. B 659, 703 (2008)

15. A. Linde. arXiv: 1402.0526 [hep-th]

16. D.S. Gorbunov, A.G. Panin, Phys. Lett. B 743, 79 (2015)

17. W.E. East, M. Kleban, A. Linde, L. Senatore, JCAP 1609(09), 010 (2016)

18. K. Clough, E.A. Lim, B.S. DiNunno, W. Fischler, R. Flauger, S. Paban, JCAP 1709(09), 025 (2017)

19. M. Kleban, L. Senatore, JCAP 1610(10), 022 (2016)

20. A.H. Guth, D.I. Kaiser, Y. Nomura, Phys. Lett. B 733, 112 (2014)

21. I. Dalianis, F. Farakos, JCAP 1507(07), 044 (2015)

22. A.D. Linde, JCAP 0410, 004 (2004)

23. Y. Hamada, H. Kawai, K.Y. Oda, S.C. Park, Phys. Rev. D 91, 053008 (2015)

24. J.J.M. Carrasco, R. Kallosh, A. Linde, Phys. Rev. D 92(6), 063519 (2015)

25. M. Artymowski, Z. Lalak, M. Lewicki. arXiv:1607.01803 [astroph.CO]

26. K. Dimopoulos, M. Artymowski, Astropart. Phys. 94, 11 (2017)

27. M. Artymowski, Z. Lalak, JCAP 1409, 036 (2014) 
28. D. Müller, A. Ricciardone, A.A. Starobinsky, A. Toporensky, Eur. Phys. J. C 78(4), 311 (2018)

29. V. Muller, H.J. Schmidt, A.A. Starobinsky, Class. Quantum Gravity 7, 1163 (1990)

30. E. Bianchi, C. Rovelli, Phys. Rev. D 84, 027502 (2011)

31. S.L. Woronowicz, Publ. RIMS Kyoto Univ. 23, 117 (1987)

32. C.A.P. Bengaly, A. Bernui, J.S. Alcaniz, I.S. Ferreira, Mon. Not. R. Astron. Soc. 466(3), 2799 (2017). arXiv:1511.09414 [astro-ph.CO]

33. V.A. Belinsky, I.M. Khalatnikov, E.M. Lifshitz, Adv. Phys. 19, 525 (1970)

34. V.A. Belinsky, I.M. Khalatnikov, E.M. Lifshitz, Adv. Phys. 31, 639 (1982)

35. J. Magueijo, L. Smolin, C.R. Contaldi, Class. Quantum Gravity 24, $3691(2007)$

36. P.C.W. Davies, Class. Quantum Gravity 5, 1349 (1988)

37. T.M. Davis, P.C.W. Davies, C.H. Lineweaver, Class. Quantum Gravity 20, 2753 (2003)

38. T.K. Mathew, R. Aiswarya, V.K. Soman, Eur. Phys. J. C 73, 2619 (2013)

39. J.D. Bekenstein, Phys. Rev. D 7, 2333 (1973)

40. S.W. Hawking, Commun. Math. Phys. 43, 199 (1975) Erratum: [Commun. Math. Phys. 46 (1976) 206]

41. G. 't Hooft, Conf. Proc. C 930308, 284 (1993)
42. J.A. Wheeler, Information, physics, quantum: the search for links, in Proceedings III International Symposium on Foundations of Quantum Mechanics, Tokyo, p. 354-368 (1989)

43. I. Prigogine, Time, structure and fluctuations, Nobel Lectures in Chemistry 1971-1980, 1993

44. M.D. Pollock, T.P. Singh, Class. Quantum Gravity 6, 901 (1989)

45. A. Barrau, L. Linsefors, JCAP 1412, 037 (2014)

46. K.A. Meissner, Class. Quantum Gravity 21, 5245 (2004)

47. P.A.R. Ade et al. [Planck Collaboration], Astron. Astrophys. 594, A13 (2016)

48. M. Mandrysz, J. Mielczarek, The top-down complexity, arXiv:1703.03301 [physics.pop-ph]

49. A. Ashtekar, Phys. Rev. Lett. 57, 2244 (1986)

50. C. Rovelli, L. Smolin, Nucl. Phys. B 331, 80 (1990)

51. J.W. Barrett, I. Naish-Guzman, Class. Quantum Gravity 26, 155014 (2009)

52. S. Mizoguchi, T. Tada, Phys. Rev. Lett. 68, 1795 (1992)

53. C. Rovelli, F. Vidotto, Phys. Rev. D 91(8), 084037 (2015) 